\title{
Estimating Change Point in Single Server Queues
}

\author{
Sibanee Sahu*, Sarat Kumar Acharya \\ P. G. Department of Statistics, Sambalpur University, Odisha, India
}

Copyright (C) 2016 by authors, all rights reserved. Authors agree that this article remains permanently

open access under the terms of the Creative Commons Attribution License 4.0 International License

\begin{abstract}
The paper is concerned with the study of change point problem in the inter-arrival time and service time of single server queues. Maximum likelihood estimators of the parameters are derived. A test statistics has been developed and its properties have been studied.
\end{abstract}

Keywords Single Server Queue, Maximum Likelihood Estimator, Change Point, Likelihood Ratio

\section{Introduction}

Statistical inference plays a major role in any use of a queueing models in decision making. Many authors have studied the parameter estimation problem in queueing models. Basawa and Prabhu[4,5] have discussed moment and likelihood estimation of the model parameters for single server queues using various sampling plans. Bhat and Rao [6] have provided an exhaustive survey of results on inference for queueing systems. Basawa and Bhat [2] studied sequential inference for the parameters of a $G I / G / 1$ queue. An empirical Bayes approach was used for estimating by Thiruvaiyaru and Basawa [15]. Basawa et al. [3] presented a maximum likelihood method for estimating the parameters of the arrival and service time distribution using only the information on the waiting times of customers in $G I / G / 1$ queue with "first come first served" queue disciline. Clarke [10] obtained the maximum likelihood estimates for the arrival and service parameters of an $M / M / 1$ queue. A review of the literature on the subject reveals that so far only single server queues have been considered from an inferential viewpoint. crane and lemoine [14] have applied simulation techniques to the problem of estimating the steady state mean waiting time in a single server queue. Acharya [1] have discussed the rate of convergence of the distribution of the maximum likelihood estimators of the arrival and the service rates in a GI/G/1 model.

Change is a natural phenomenon which occurs in every sphere of works. In statistics we are interested in the statistical analysis of change point detection and estimation.

Let $X_{1}, X_{2}, X_{3}, \ldots, X_{n}$ be a sequence of independent random variables with probability distribution functions $F_{1}, F_{2}, F_{3}, \ldots, F_{n}$, respectively.

Then, in general, the change point problem is to test the following null hypothesis:

$H_{0}: F_{1}=F_{2}=\ldots=F_{n} \quad$ verses the alternative hypothesis

$H_{0}: F_{1}=F_{2}=\ldots=F_{k} \neq F_{k+1}=\ldots=F_{n}$

If the distributions $F_{1}, F_{2}, F_{3}, \ldots, F_{n}$ belongs to the common parametric family $F(\theta)$, where $\theta \in R^{p}$, then the change point problem is to test the hypothesis about the population parameter $\theta_{i}, i=1,2,3, \ldots, n$,

$$
\begin{aligned}
& H_{0}: \theta_{1}=\theta_{2}=\ldots=\theta_{n}=\theta(\text { unknown }) \quad \text { verses the alternative hypothesis } \\
& H_{0}: \theta_{1}=\theta_{2}=\ldots=\theta_{k} \neq \theta_{k+1}=\ldots=\theta_{n}
\end{aligned}
$$

The problem of testing of parameter change has long been a core issue in statistical inferences. It originally started in the quality control context and then rapidly moved to various areas such as economics, finance, transportation systems, statistical quality control, inventory, production processes, communication networks and queueing, control problems, medicine. The change point problem was first dealt in independent and identically distributed samples but it became very popular in time 
series models. For relevant references in i.i.d samples and time series models, we refer to Brown, Durbin and Evans[7], Wichern, Miller and Hsu[17], Zacks[18], Krishnaiah and Miao[13] and the references therein.

The problem of estimating change point of the inter-arrival time distribution in the queueing is of great interest. Besides maximum likelihood and least square estimates, the Bayesian method is also a very useful technique for estimating parameters. Chernoff and Zacks [8] has studied the change point problem using the Bayesian method.

The main goal of this paper is to study the change point problem for the single server queue.

In section 2 preliminary results about the maximum likelihood estimators of a GI/G/1 queue have been mentioned. Section 3 deals with the change point estimation for the interarrival time distribution of the GI/G/1 queue . A test statistic has been developed.

\section{The GI/G/1 Queue}

Consider a single server queueing system in which the interarrival times $\left\{u_{k}, k \geq 1\right\}$ and the service times $\left\{v_{k}, k \geq 1\right\}$ are two independent sequences of independent and identically distributed nonnegative random variables with densities $f(u ; \theta)$ and $g(v ; \phi)$, respectively, where $\theta$ and $\phi$ are unknown parameters. Let us assume that $f$ and $g$ belong to the continuous exponential families given by

$$
\begin{aligned}
& f(u ; \theta)=a_{1}(u) e^{\left[\theta h_{1}(u)-k_{1}(\theta)\right]} \\
& g(v ; \phi)=a_{2}(v) e^{\left[\phi h_{2}(v)-k_{2}(\phi)\right]}
\end{aligned}
$$

It is further assumed that the densities in (1) and in (2) are equal to Zero on $(-\infty, 0)$.

For simplicity we assume that the initial customer arrives at time $t=0$. Our sampling scheme is to observe the system over a continuous time interval $[0, T]$ where $T$ is a suitable stopping time. The sample data consist of

$$
A(T), D(T), u_{1}, u_{2}, u_{3}, \cdots \cdots, u_{A(T)}, v_{1}, v_{2}, \cdots \cdots, v_{D(T)}
$$

where $A(T)$ is the number of arrivals and $D(T)$ is the number of departures during $(0, T]$. Obviously no arrivals occur during $\left[\sum_{i=1}^{A(T)} u_{i}, T\right]$ and no departures during $\left[\gamma(T)+\sum_{i=1}^{D(T)} v_{i}, T\right]$, where $\gamma(T)$ is the total idle period in $(0, T]$.

some possible stopping rules to determine $T$ are given below:

Rule 1. Observe the system until a fixed time $t$. Here $T=t$ with probability one and $A(T)$ and $D(T)$ are both random variables.

Rule 2. Observe the system until $d$ departures have occured so that $D(T)=d$. Here $T=\gamma(T)+v_{1}+v_{2}+\cdots+v_{d}$ and $A(T)$ are random variables.

Rule 3. Observe the system until $m$ arrivals take place so that $A(T)=m$. Here $T=u_{1}+u_{2}+u_{3}+\cdots+u_{m}$ and $D(T)$ are random variables.

Rule 4. Stop at the $n t h$ transition epoch. Here, $T, A(T)$ and $D(T)$ are all random variables and $A(T)+D(T)=n$.

Under rule 4, we stop either with an arrival or in a departure. If we stop with an arrival, then $\sum_{i=1}^{A(T)} u_{i}=T$ and no departures during $\left[\gamma(T)+\sum_{i=1}^{D(T)} v_{i}, T\right]$. Similarly, if we stop in a departure, then $\gamma(T)+\sum_{i=1}^{D(T)} v_{i}=T$ and there are no arrivals during $\left[\sum_{i=1}^{A(T)} u_{i}, T\right]$.

The likelihood function based on data (3) is given by

$$
\begin{aligned}
L_{T}(\theta, \phi) & =\prod_{i=1}^{A(T)} f\left(u_{i}, \theta\right) \prod_{i=1}^{D(T)} f\left(v_{i}, \phi\right) \\
& \times\left[1-F_{\theta}\left[T-\sum_{i=1}^{A(T)} u_{i}\right]\right]\left[1-G_{\phi}\left[T-\gamma(T)-\sum_{i=1}^{D(T)} v_{i}\right]\right]
\end{aligned}
$$

where $F$ and $G$ are distribution functions corresponding to the densities $f$ and $g$, respectively. The likelihood function $L_{T}(\theta, \phi)$ remains valid under all the stopping rules.

The approximate likelihood $L_{T}^{a}(\theta, \phi)$ is defined as

$$
L_{T}^{a}(\theta, \phi)=\prod_{i=1}^{A(T)} f\left(u_{i}, \theta\right) \prod_{i=1}^{D(T)} f\left(v_{i}, \phi\right)
$$


The maximum likelihood estimates obtained from (5) are asymptotically equivalent to those obtained from (4) provided the following two conditions are satisfied as $T \rightarrow \infty$ :

$$
\begin{gathered}
A(T)^{-\frac{1}{2}} \frac{\partial}{\partial \theta} \ln \left[1-F_{\theta}\left[T-\sum_{i=1}^{A(T)} u_{i}\right]\right] \underline{p} \quad 0 \\
D(T)^{-\frac{1}{2}} \frac{\partial}{\partial \phi} \ln \left[1-G_{\phi}\left[T-\gamma(T)-\sum_{i=1}^{D(T)} v_{i}\right]\right] \underline{p} \quad 0
\end{gathered}
$$

\subsection{Approximate maximum Likelihood Estimates}

The interarrival time density $f(u ; \theta)$ and the service time density $g(v ; \phi)$ belongs to exponential families given by (1) and (2). It is easily verified that the moment generating function of the random variables $h_{1}(u)$ and $h_{2}(v)$ is given by $M(t)=\exp \left[k_{1}(t+\theta)-k_{1}(\theta)\right]$ and $M(t)=\exp \left[k_{2}(t+\phi)-k_{2}(\phi)\right]$ respectively. Consequently

$$
\begin{array}{ll}
\eta_{1}(\theta)=\mathrm{E}_{\theta}\left[h_{1}(u)\right]=k_{1}^{\prime}(\theta), & \sigma_{1}^{2}=\operatorname{Var}_{\theta}\left[h_{1}(u)\right]=k_{1}^{\prime \prime}(\theta) \\
\eta_{2}(\phi)=\mathrm{E}_{\phi}\left[h_{2}(v)\right]=k_{2}^{\prime}(\phi), & \sigma_{2}^{2}=\operatorname{Var}_{\phi}\left[h_{2}(v)\right]=k_{2}^{\prime \prime}(\phi)
\end{array}
$$

The approximate likelihood function $L_{T}^{a}(\theta, \phi)$ is reduced to

$$
\begin{aligned}
L_{T}^{a}(\theta, \phi) & =\prod_{i=1}^{A(T)} a_{1}\left(u_{i}\right) \prod_{i=1}^{D(T)} a_{2}\left(v_{i}\right) \\
& \times \exp \left(\sum_{i=1}^{A(T)}\left[\theta h_{1}\left(u_{i}\right)-k_{1}(\theta)\right]+\sum_{i=1}^{D(T)}\left[\phi h_{2}\left(v_{i}\right)-k_{2}(\phi)\right]\right)
\end{aligned}
$$

and the $\log$ likelihood function becomes

$$
\begin{aligned}
l(\theta, \phi)^{a}= & \log L_{T}^{a}(\theta, \phi) \\
= & \log \left[\prod_{i=1}^{A(T)} a_{1}\left(u_{i}\right) \prod_{i=1}^{D(T)} a_{2}\left(v_{i}\right)\right]+\sum_{i=1}^{A(T)}\left[\theta h_{1}\left(u_{i}\right)-k_{1}(\theta)\right] \\
& +\sum_{i=1}^{D(T)}\left[\phi h_{2}\left(v_{i}\right)-k_{2}(\phi)\right]
\end{aligned}
$$

Differentiating $l(\theta, \phi)^{a}$ w.r.t $\theta$ and $\phi$ and then equating to zero, we get

$$
\frac{\partial}{\partial \theta} l(\theta, \phi)^{a}=\sum_{i=1}^{A(T)} h_{1} u_{i}-A(T) k_{1}^{\prime}(\theta)=0
$$

and

$$
\frac{\partial}{\partial \phi} l(\theta, \phi)^{a}=\sum_{i=1}^{D(T)} h_{2} v_{i}-D(T) k_{2}^{\prime}(\phi)=0
$$

From now on we shall write $l$ for $L_{T}^{a}$. The estimating equation reduce to

$$
\begin{aligned}
& k_{1}^{\prime}(\theta)=\frac{\sum_{i=1}^{A(T)} h_{1}\left(u_{i}\right)}{A(T)}=\eta_{1}(\theta) \\
& k_{2}^{\prime}(\phi)=\frac{\sum_{i=1}^{D(T)} h_{2}\left(v_{i}\right)}{D(T)}=\eta_{2}(\phi)
\end{aligned}
$$

The solution for $\theta$ and $\phi$ from (14) and (15) are given by

$$
\hat{\theta}=\eta_{1}^{-1}\left[(A(T))^{-1} \sum_{i=1}^{A(T)} h_{1} u_{i}\right], \quad \hat{\phi}=\eta_{2}^{-1}\left[(D(T))^{-1} \sum_{i=1}^{D(T)} h_{2} v_{i}\right]
$$




\section{Change Point Problem of the Inter-arrival Time}

Let's consider the $G I / G / 1$ queueing system in which interarrival times $u_{k}, k \geq 1$ and the service times $v_{k}, k \geq 1$ are the two independent sequence of independent and identically distributed non-negative random variables with density $f(u, \theta)$ and $g(v, \phi)$ respectively.

We are interested in testing the null hypothesis that $u_{1}, u_{2}, \cdots \cdots, u_{A(T)}$ are i.i.d from exponential distribution with parameter $\theta_{0}$ against the alternative hypothesis that at some point $l$ a change occurs in parameter $\theta$ i.e. for some $l \in$ $1,2,3, \cdots \cdots, A(T)-1, u_{1}, u_{2}, \cdots \cdots, u_{l}$ are i.i.d from exponential distribution with parameter $\theta_{0}$ and $u_{l+1}, u_{l+2}, \cdots \cdots, u_{A(T)}$ are i.i.d from exponential distribution with parameter $\theta_{0}+\delta$.

We can write this as

$$
\begin{aligned}
& H_{0}: \theta_{1}=\theta_{2}=\cdots \cdots=\theta_{A(T)}=\theta_{0} \quad \text { Against } \\
& H_{1}: \theta_{1}=\theta_{2}=\cdots \cdots=\theta_{l}=\theta_{0} \neq \theta_{l+1}=\cdots \cdots=\theta_{A(T)}=\left(\theta_{0}+\delta\right)
\end{aligned}
$$

Following Kander and Zacks[11] we derive a test statistic using quasi Bayesian approach by considering the point of change $l$ as a realization of a random variable $L$ with a uniform prior density.

$$
\pi(l)= \begin{cases}\frac{1}{A(T)-1}, & l=1,2,3, \ldots, A(T)-1 \\ 0, & \text { otherwise }\end{cases}
$$

The loglikelihood function under the null hypothesis is given by

$$
\begin{aligned}
& l_{0}\left(\theta_{0}, \phi\right)^{a}=\log \left[\prod_{i=1}^{A(T)} a_{1}\left(u_{i}\right) \prod_{i=1}^{D(T)} a_{2}\left(v_{i}\right)\right]+\sum_{i=1}^{A(T)}\left[\theta_{0} h_{1}\left(u_{i}\right)-k_{1}\left(\theta_{0}\right)\right] \\
& +\sum_{i=1}^{D(T)}\left[\phi h_{2}\left(v_{i}\right)-k_{2}(\phi)\right] \\
& \Rightarrow \frac{\partial l_{0}\left(\theta_{0}, \phi\right)^{a}}{\partial \theta_{0}}=0 \\
& \Rightarrow \frac{\partial}{\partial \theta_{0}} \sum_{i=1}^{A(T)}\left[\theta_{0} h_{1}\left(u_{i}\right)-k_{1}\left(\theta_{0}\right)\right]=0 \\
& \Rightarrow k_{1}\left(\theta_{0}\right)=\frac{\sum_{i=1}^{A(T)} h_{1}\left(u_{i}\right)}{A(T)} \\
& \left.\Rightarrow \hat{\theta_{0}}=\eta_{1}^{-1}\left[(A(T))^{-1} \sum_{i=1}^{A(T)} h_{1}\left(u_{i}\right)\right]\right]
\end{aligned}
$$

Under the alternative hypothesis

$$
\begin{aligned}
L_{1}\left(\theta_{0}, \phi\right)^{a} & =\left\{\sum_{l=1}^{A(T)-1} \pi(l)\left(\prod_{i=1}^{l} a_{1}\left(u_{i}\right) e^{\left[\theta_{0} h_{1}\left(u_{i}\right)-k_{1}\left(\theta_{0}\right)\right]}\right)\left(\prod_{i=l+1}^{A(T)} a_{1}\left(u_{i}\right) e^{\left[\left(\theta_{0}+\delta\right) h_{1}\left(u_{i}\right)-k_{1}\left(\theta_{0}+\delta\right)\right]}\right)\right\} \\
& {\left[\prod_{i=1}^{D(T)} a_{2}\left(v_{i}\right) e^{\left[\phi h_{2}\left(v_{i}\right)-k_{2}(\phi)\right]}\right] } \\
& =\frac{1}{A(T)-1} \sum_{l=1}^{A(T)-1}\left\{\exp \left[\sum_{i=1}^{l}\left(\log a_{1}\left(u_{i}\right)+\theta_{0} h_{1}\left(u_{i}\right)-k_{1}\left(\theta_{0}\right)\right)\right]\right. \\
& \left.+\left[\sum_{i=l+1}^{A(T)}\left(\log a_{1}\left(u_{i}\right)+\left(\theta_{0}+\delta\right) h_{1}\left(u_{i}\right)-k_{1}\left(\theta_{0}+\delta\right)\right)\right]\right\}\left[\prod_{i=1}^{D(T)} a_{2}\left(v_{i}\right) e^{\left[\phi h_{2}\left(v_{i}\right)-k_{2}(\phi)\right]}\right]
\end{aligned}
$$

Now by taylor expansion

$$
\begin{aligned}
& \log a_{1}\left(u_{i}\right)+\left(\theta_{0}+\delta\right) h_{1}\left(u_{i}\right)-k_{1}\left(\theta_{0}+\delta\right) \\
& =\log a_{1}\left(u_{i}\right)+\theta_{0} h_{1}\left(u_{i}\right)-k_{1}\left(\theta_{0}\right)+\delta\left[h_{1}\left(u_{i}\right)-k_{1}^{\prime}\left(\theta_{0}\right)\right]+o(\delta), \quad \text { as } \quad \delta \rightarrow 0
\end{aligned}
$$


Now

$$
\begin{aligned}
& L_{1}\left(\theta_{0}, \phi\right)^{a}=\frac{1}{A(T)-1} \sum_{l=1}^{A(T)-1}\left\{\operatorname { e x p } \left[\sum_{i=1}^{l}\left(\log a_{1}\left(u_{i}\right)+\theta_{0} h_{1}\left(u_{i}\right)-k_{1}\left(\theta_{0}\right)\right)\right.\right. \\
& \left.\left.+\sum_{i=l+1}^{A(T)}\left(\log a_{1}\left(u_{i}\right)+\theta_{0} h_{1}\left(u_{i}\right)-k_{1}\left(\theta_{0}\right)\right)+\sum_{i=l+1}^{A(T)} \delta\left[h_{1}\left(u_{i}\right)-k_{1}^{\prime}\left(\theta_{0}\right)\right]+o(\delta)\right]\right\} \\
& {\left[\prod_{i=1}^{D(T)} a_{2}\left(v_{i}\right) e^{\left[\phi h_{2}\left(v_{i}\right)-k_{2}(\phi)\right]}\right]} \\
& =\frac{1}{A(T)-1} \sum_{l=1}^{A(T)-1}\left\{\operatorname { e x p } \left[\sum_{i=1}^{A(T)}\left(\log a_{1}\left(u_{i}\right)+\theta_{0} h_{1}\left(u_{i}\right)-k_{1}\left(\theta_{0}\right)\right)\right.\right. \\
& \left.\left.+\sum_{i=l+1}^{A(T)} \delta\left[h_{1}\left(u_{i}\right)-k_{1}^{\prime}\left(\theta_{0}\right)\right]+o(\delta)\right]\right\}\left[\prod_{i=1}^{D(T)} a_{2}\left(v_{i}\right) e^{\left[\phi h_{2}\left(v_{i}\right)-k_{2}(\phi)\right]}\right] \\
& =\left[\prod_{i=1}^{A(T)} a_{1}\left(u_{i}\right) e^{\left[\theta h_{1}\left(u_{i}\right)-k_{1}(\theta)\right]}\right]\left[\prod_{i=1}^{D(T)} a_{2}\left(v_{i}\right) e^{\left[\phi h_{2}\left(v_{i}\right)-k_{2}(\phi)\right]}\right] \\
& \left\{\frac{1}{A(T)-1} \sum_{l=1}^{A(T)-1} \exp \left[\sum_{i=l+1}^{A(T)} \delta\left[h_{1}\left(u_{i}\right)-k_{1}^{\prime}\left(\theta_{0}\right)\right]+o(\delta)\right]\right\} \\
& =\left[L_{0}\left(\theta_{0}, \phi\right)^{a}\right]\left[\sum_{l=1}^{A(T)-1} \frac{1}{A(T)-1}\left(1+\sum_{i=l+1}^{A(T)} \delta\left[h_{1}\left(u_{i}\right)-k_{1}^{\prime}\left(\theta_{0}\right)\right]+o(\delta)\right)\right] \\
& =\left[L_{0}\left(\theta_{0}, \phi\right)^{a}\right]\left[1+\frac{\delta}{A(T)-1} \sum_{l=1}^{A(T)-1} \sum_{i=l+1}^{A(T)}\left[h_{1}\left(u_{i}\right)-k_{1}^{\prime}\left(\theta_{0}\right)\right]+o(\delta)\right] \\
& =\left[L_{0}\left(\theta_{0}, \phi\right)^{a}\right]\left[1+\frac{\delta}{A(T)-1} \sum_{i=1}^{A(T)}(i-1)\left[h_{1}\left(u_{i}\right)-k_{1}^{\prime}\left(\theta_{0}\right)\right]+o(\delta)\right] \quad \text { as } \quad \delta \rightarrow 0
\end{aligned}
$$

The likelihood ratio is

$$
\begin{aligned}
\Lambda & =\frac{L_{1}\left(\theta_{0}, \phi\right)^{a}}{L_{0}\left(\theta_{0}, \phi\right)^{a}} \\
& =\left[1+\frac{\delta}{A(T)-1} \sum_{i=1}^{A(T)}(i-1)\left[h_{1}\left(u_{i}\right)-k_{1}^{\prime}\left(\theta_{0}\right)\right]+o(\delta)\right] \quad \text { as } \quad \delta \rightarrow 0
\end{aligned}
$$

Now our test statistic is

$$
T_{A(T)}=\sum_{i=1}^{A(T)}(i-1)\left(h_{1}\left(u_{i}\right)-k_{1}^{\prime}\left(\theta_{0}\right)\right)
$$

The exact distribution of $T_{A(T)}$ is obtained as follows

$$
E\left[T_{A(T)}\right]=E\left[\sum_{i=1}^{A(T)}(i-1)\left(h_{1}\left(u_{i}\right)-k_{1}^{\prime}\left(\theta_{0}\right)\right)\right]=0
$$

(Since $h_{1}\left(u_{i}\right)$ 's are exponential under null hypothesis)

$$
\begin{aligned}
\operatorname{Var}\left[T_{A(T)}\right]= & \operatorname{var}\left[\sum_{i=1}^{A(T)}(i-1)\left(h_{1}\left(u_{i}\right)-k_{1}^{\prime}\left(\theta_{0}\right)\right)\right] \\
& =\frac{E[A(T)(A(T)-1)(2 A(T)-1)]}{6} \cdot k_{1}^{\prime \prime}\left(\theta_{0}\right)
\end{aligned}
$$




$$
=\frac{E[A(T)(A(T)-1)(2 A(T)-1)]}{6} \cdot \sigma_{1}^{2}\left(\theta_{0}\right)
$$

The test statistics $T_{A(T)}$ follows gamma distribution with mean 0 and variance $\frac{E\{A(T)(A(T)-1)(2 A(T)-1)\}}{6} \cdot \sigma_{1}^{2}\left(\theta_{0}\right)$

Similarly we can show that when there is a change in parameter $\phi$, then the test statistics is

$$
T_{D(T)}=\sum_{i=1}^{D(T)}(i-1)\left(h_{2}\left(v_{i}\right)-k_{2}^{\prime}\left(\phi_{0}\right)\right)
$$

The test statistics $T_{D(T)}$ follows gamma distribution with mean 0 and variance $\frac{E\{D(T)(D(T)-1)(2 D(T)-1)\}}{6} \cdot \sigma_{2}^{2}\left(\phi_{0}\right)$

\section{Example}

Let's consider the $M / M / 1$ queueing system with a poisson arrival and exponential service time .Let the interarrival times $u_{i}, i \geq 1$ and the service times $v_{i}, i \geq 1$ are two independent sequence of independent and identically distributed non-negative random variables with density $f(u, \lambda)$ and $g(v, \mu)$ respectively given as

$$
\begin{array}{llrl}
f(u, \lambda) & =\lambda e^{-\lambda u_{i}}, & & u_{i}>0 \\
f(v, \mu) & =\mu e^{-\mu v_{i}}, & & v_{i}>0
\end{array}
$$

We are interested in testing the null hypothesis that $u_{1}, u_{2}, \cdots, \cdots, u_{A(T)}$ are i.i.d from exponential distribution with parameter $\lambda_{0}$ against the alternative hypothesis that at some point $l$ a change occurs in parameter $\lambda$ i.e. for some $l \in$ $1,2,3, \cdots, A(T)-1, u_{1}, u_{2}, \cdots \cdots, u_{l}$ are i.i.d from exponential distribution with parameter $\lambda_{0}$ and $u_{l+1}, u_{l+2}, \cdots \cdots, u_{A(T)}$ are i.i.d from exponential distribution with parameter $\left(\lambda_{0}+\delta\right)$.

We can write this as

$$
\begin{aligned}
& H_{0}: \lambda_{1}=\lambda_{2}=\lambda_{3}=\ldots=\lambda_{A(T)}=\lambda_{0} \quad \text { against } \\
& H_{1}: \lambda_{1}=\lambda_{2}=\lambda_{3}=\ldots=\lambda_{l}=\lambda_{0} \neq \lambda_{l+1}=\ldots=\lambda_{A(T)}=\left(\lambda_{0}+\delta\right)
\end{aligned}
$$

Then the likelihood function under the null hypothesis can be obtained as

$$
\begin{aligned}
L_{0}\left(\lambda_{0}, \mu\right) & =\left(\prod_{i=1}^{A(T)} \lambda_{0} e^{-\lambda_{0} u_{i}}\right)\left(\prod_{i=1}^{D(T)} \mu e^{-\mu v_{i}}\right) \\
& =\left[\lambda_{0}^{A(T)} e^{\left(-\lambda_{0} \sum_{i=1}^{A(T)} u_{i}\right)}\right]\left[\mu^{D(T)} e^{\left(-\mu \sum_{i=1}^{D(T)} v_{i}\right)}\right]
\end{aligned}
$$

and the maximum likelihood estimator of $\lambda$ is

$$
\hat{\lambda_{0}}=\frac{\sum_{i=1}^{A(T)} u_{i}}{A(T)}
$$

Under the alternative hypothesis the likelihood function

$$
\begin{aligned}
L_{1}\left(\lambda_{0}, \mu\right)^{a} & =\left\{\sum_{l=1}^{A(T)-1} \pi(l)\left(\prod_{i=1}^{l} \lambda_{0} e^{-\lambda_{0} u_{i}}\right)\left(\prod_{i=l+1}^{A(T)}\left(\lambda_{0}+\delta\right) e^{\left(\lambda_{0}+\delta\right) u_{i}}\right)\right\}\left\{\prod_{i=1}^{D(T)} \mu e^{-\mu v_{i}}\right\} \\
& =\left[L_{0}\left(\lambda_{0}, \mu\right)^{a}\right]\left[1+\frac{\delta}{A(T)-1} \sum_{i=1}^{A(T)}(i-1)\left(\frac{1}{\lambda_{0}}-u_{i}\right)+o(\delta)\right] \quad \text { as } \quad \delta \rightarrow 0
\end{aligned}
$$

The likelihood ratio is

$$
\Lambda=\left[1+\frac{\delta}{A(T)-1} \sum_{i=1}^{A(T)}(i-1)\left(\frac{1}{\lambda_{0}}-u_{i}\right)+o(\delta)\right] \quad \text { as } \quad \delta \rightarrow 0
$$

Now our test statistic is

$$
T_{A(T)}=\sum_{i=1}^{A(T)}(i-1)\left(\frac{1}{\lambda_{0}}-u_{i}\right)+o(\delta)
$$


The exact distribution of $T_{A(T)}$ is obtained as follows

$$
\begin{gathered}
E\left[T_{A(T)}\right]=\quad E\left[\sum_{i=1}^{A(T)}(i-1)\left(\frac{1}{\lambda_{0}}-u_{i}\right)+o(\delta)\right]=0 \\
\operatorname{Var}\left[T_{A(T)}\right] \quad=\operatorname{Var}\left[\sum_{i=1}^{A(T)}(i-1)\left(\frac{1}{\lambda_{0}}-u_{i}\right)+o(\delta)\right] \\
=\frac{E[A(T)(A(T)-1)(2 A(T)-1)]}{6} \cdot \frac{1}{\lambda_{0}^{2}}
\end{gathered}
$$

The test statistics $T_{A(T)}$ follows gamma distribution with mean 0 and variance $\frac{E[A(T)(A(T)-1)(2 A(T)-1)]}{6} \frac{1}{\lambda_{0}^{2}}$ Similarly we can show that when there is a change in parameter $\mu$, then the test statistics is

$$
T_{D(T)}=\sum_{i=1}^{D(T)}(i-1)\left(\frac{1}{\mu_{0}}-v_{i}\right)
$$

The test statistics $T_{D(T)}$ follows gamma distribution with mean 0 and variance $\frac{E\{D(T)(D(T)-1)(2 D(T)-1)\}}{6} \frac{1}{\mu_{0}^{2}}$

\subsection{Simulation}

For a $M / M / 1$ queue we generated $A(T)$ observations $u_{1}, u_{2}, \cdots, u_{A(T)}$ and $D(T)$ observations $v_{1}, v_{2}, \cdots, \cdots, v_{D(T)}$ For both $A(T)$ and $D(T)$ takes value $100,500,1000,1500,2000$ with different values of $\lambda_{0}=0.5,0.8,1.5,2.8,1.6$ and $\mu_{0}=0.6,0.9,2.5,1.7,2.1$ respectively. The estimated value of $\lambda_{0}$ and $\mu_{0}$ was computed using maximum likelihood estimation method. When there is a change occurs in parameter $\lambda$ and $\mu$ at some point $l$, the test statistic and it's mean and variance value are derived. The simulation results are given in the Table- 1 and Table- 2 respectively.

Table 1. When change in parameter $\lambda$

\begin{tabular}{|c|c|c|c|c|c|}
\hline$A(T)$ & $\lambda_{0}$ & $\hat{\lambda}_{0}$ & $T_{A(T)}$ & $E\left(T_{A(T)}\right)$ & $V\left(T_{A(T)}\right)$ \\
\hline 100 & 0.5 & 1.908228 & -7134.719 & 0 & 90172.99 \\
\hline 500 & 0.8 & 1.244679 & -57621.76 & 0 & 26814522 \\
\hline 1000 & 1.5 & 0.6598723 & 431380.6 & 0 & 764376340 \\
\hline 1500 & 2.8 & 0.3449658 & 2880042 & 0 & 9444218472 \\
\hline 2000 & 1.6 & 0.6113597 & 4576490 & 0 & 22391895633 \\
\hline
\end{tabular}

Table 2. When change in parameter $\mu$

\begin{tabular}{|c|c|c|c|c|c|}
\hline$D(T)$ & $\mu_{0}$ & $\hat{\mu_{0}}$ & $T_{D(T)}$ & $E\left(T_{D(T)}\right)$ & $V\left(T_{D(T)}\right)$ \\
\hline 100 & 0.6 & 1.581461 & -3914.184 & 0 & 131286.5 \\
\hline 500 & 0.9 & 1.093193 & -14916.91 & 0 & 34760907 \\
\hline 1000 & 2.5 & 0.3866171 & 1094239 & 0 & 2226716433 \\
\hline 1500 & 1.7 & 0.598574 & 1212004 & 0 & 3136768081 \\
\hline 2000 & 2.1 & 0.4790627 & 2400702 & 0 & 7437162079 \\
\hline
\end{tabular}

\section{Acknowledgments}

The work is supported by University Grants Commission under the head "MajorResearchProject".

\section{REFERENCES}

[1] Acharya, S.K (1999). On normal approximation for maximum likelihood estimation from single server queues, Queueing System. 31, $207-216$.

[2] Basawa, I.V. and Bhat, B.R. (1992). Sequential inference for single server queues, Queueing and related models. Eds, U.N.Bhat and I.V.Basawa, Oxford University press Oxford. $325-336$. 
[3] Basawa, I.V. Bhat, U.N and Robert Lund (1996). Maximum likelihood estimation for single server queues from waiting time data, Queueing Systems. 24, $155-167$

[4] Basawa, I.V. and Prabhu, N.U. (1981). Estimation in single server queues, Naval Res. Logist. 28, 475 - 487.

[5] Basawa, I.V. and Prabhu, N.U. (1988). Large sample inference from single server queues, Queueing Systems. 3, $288-304$

[6] Bhat,U.N. and Rao, S.S. (1987). Statistical Analysis of Queueing Systems, Queueing Systems. 1, 217 - 247

[7] Brown, R.L., Durbin, J., and evans, J. M. (1975). Tcheniques for testing the constancy of regression relationships over time with disscussion, Journal of the Royal Statistical Society B.37,149 - 192.

[8] Chernoff, H. and Zacks, S. (1964). Estimating the current mean of a normal distribution which is subject to changes in time, Annals of Mathematical Statistics. 35, $999-1018$.

[9] Chen, J and Gupta, A.K. (2000). Parametric statistical change point analysis, Birkhäuser, Boston.

[10] Clerke, A. B. (1957). Maximum Likelihood Estimates in a simple queue, Annal. Math. Stat. 28, 1036 - 1040.

[11] Kander, Z. and Zacks, S. (1966). Test Procedures for possible changes in parameters of Statistical distributions occuring at unknown time points Annals of Mathematical Statistics, 37, 1196 - 1210.

[12] Kelly,F.P (1979).Reversibility and Stochastic Networks, Wiley, New York

[13] Krishnaiah, P. R. and Miao,B.Q (1988).Review about estimation of change points (P.R. krishnaiah and C.R.Rao, Eds.) handbook of statistics.7, Elsevier,Amsterdam $375-402$

[14] Lemoine, A. J. (1977) Networks of Queue- A Survey of Equilibrium Analysis, Manage. Sci. 24, 464 - 481

[15] Thiruvaiyaru, D and Basawa, I.V. (1991) Estimation For a Class of Simple Queueing Networks, Queueing Systems 9 , $301-312$

[16] Thiruvaiyaru, D and Basawa, I.V. (1992) Emperical bayes estimation for queueing systems and networks, Queueing Systems 11, $179-202$

[17] Wichern, D.W., Miller, R.B., and Hsu, D.A (1976) changes of varience in first order autoregressive time series models with an applications, Applied Statistics. 25, $248-356$

[18] Zacks, S. (1983) Survey of Classical and Bayesian approaches to the change Point problem: Fixed sample and sequential procedures of testing and estimation, Recent Advances in Statistics. Academic Press.245 - 269 